This is the Table of Contents for the most recent online-only supplemental issue TOMM 15(2s). Please find this supplemental issue in the ACM Digital Library and enjoy reading the issue!

Table of Contents: Online Supplement Volume 15, Number $2 \mathrm{~s}$

Special Issue on Cross-Media Analysis for Visual Question Answering

Introduction to the Special Issue on the Cross-Media Analysis for Visual Question Answering Richang Hong, Yahong Han, Tat-Seng Chua

Article 48

DOI: https://doi.org/10.1145/3337985

Semantic Concept Network and DeepWalk-based Visual Question Answering

Qun Li, Fu Xiao, Le An, Xianzhong Long, Xiaochuan Sun

Article 49

DOI: https://doi.org/10.1145/3300938

BTDP: Toward Sparse Fusion with Block Term Decomposition Pooling for Visual

Question Answering

Zhiwei Fang, Jing Liu, Xueliang Liu, Qu Tang, Yong Li, Hanqing Lu

Article 50

DOI: https://doi.org/10.1145/3282469

Multi-source Multi-level Attention Networks for Visual Question Answering

Dongfei Yu, Jianlong Fu, Xinmei Tian, Tao Mei

Article 51

DOI:https://doi.org/10.1145/3316767

Video Question Answering via Knowledge-based Progressive Spatial-Temporal Attention Network Weike Jin, Zhou Zhao, Yimeng Li, Jie Li, Jun Xiao, Yueting Zhuang

Article 52

DOI: https://doi.org/10.1145/3321505

Spatiotemporal-Textual Co-Attention Network for Video Question Answering

Zheng-Jun Zha, Jiawei Liu, Tianhao Yang, Yongdong Zhang

Article 53

DOI: https://doi.org/10.1145/3320061

Show, Reward, and Tell: Adversarial Visual Story Generation

Jinhui Tang, Jing Wang, Zechao Li, Jianlong Fu, Tao Mei

Article 54

DOI: https://doi.org/10.1145/3291925

(c) 2019 Copyright held by the owner/author(s).

1551-6857/2019/08-ART69e

https://doi.org/10.1145/3359668 
Image Captioning by Asking Questions

Xiaoshan Yang, Changsheng Xu

Article 55

DOI: https://doi.org/10.1145/3313873

Cross-Modality Retrieval by Joint Correlation Learning

Shuo Wang, Dan Guo, Xin Xu, Li Zhuo, Meng Wang

Article 56

DOI: https://doi.org/10.1145/3314577

\section{Special Issue on Big Data, Machine Learning and AI Technologies for Art and Design}

Introduction to the Special Issue on Big Data, Machine Learning, and AI Technologies for Art and Design

Prof. James She, Prof. Eugene Ch'ng, Dr. Tao Mei, Prof. Miu Ling Lam, Prof. Chee Onn Wong, Prof. Jane Prophet, Prof. Ying-Qing Xu, Prof. Byung Gook Lee, Many Anonymous Reviewers Article 57

DOI: https://doi.org/10.1145/3338002

Artificial Intelligence, Artists, and Art: Attitudes Toward Artwork Produced by Humans vs.

Artificial Intelligence

Joo-Wha Hong, Nathaniel Ming Curran

Article 58

DOI: https://doi.org/10.1145/3326337

Art by Computing Machinery: Is Machine Art Acceptable in the Artworld?

Eugene Ch'ng

Article 59

DOI: https://doi.org/10.1145/3326338

Visual Arts Search on Mobile Devices

Hui Mao, James She, Ming Cheung

Article 60

DOI: https://doi.org/10.1145/3326336

Interpretable Partitioned Embedding for Intelligent Multi-item Fashion Outfit Composition Zunlei Feng, Zhenyun Yu, Yongcheng Jing, Sai Wu, Mingli Song, Yezhou Yang, Junxiao Jiang Article 61

DOI: https://doi.org/10.1145/3326332

Color Theme-based Aesthetic Enhancement Algorithm to Emulate the Human Perception of Beauty in Photos

Karen Panetta, Long Bao, Sos Agaian, Victor Oludare

Article 62

DOI: https://doi.org/10.1145/3328991 
Beauty Is in the Eye of the Beholder: Demographically Oriented Analysis of Aesthetics in Photographs

Magzhan Kairanbay, John See, Lai-Kuan Wong

Article 63

DOI: https://doi.org/10.1145/3328993

\section{Special Section on MMSys/NOSSDAV/MMVE 2018}

Introduction to the Best Papers of the ACM Multimedia Systems (MMSys) Conference 2018 and the ACM Workshop on Network and Operating System Support for Digital Audio and Video (NOSSDAV) 2018 and the InternationalWorkshop on Mixed and Virtual Environment Systems (MMVE) 2018

Pablo Cesar, Michael Zink, Niall Murray

Article 64

DOI: https://doi.org/10.1145/3339846

Game of Streaming Players: Is Consensus Viable or an Illusion?

Abdelhak Bentaleb, Ali C. Begen, Saad Harous, Roger Zimmermann

Article 65

DOI: https://doi.org/10.1145/3336496

A Framework for Adaptive Residual Streaming for Single-Player Cloud Gaming De-Yu Chen, Magda El-Zarki

Article 66

DOI: https://doi.org/10.1145/3336498

From Theory to Practice: Improving Bitrate Adaptation in the DASH Reference Player Kevin Spiteri, Ramesh Sitaraman, Daniel Sparacio

Article 67

DOI: https://doi.org/10.1145/3336497

6K and 8K Effective Resolution with 4K HEVC Decoding Capability for 360 Video Streaming Alireza Zare, Maryam Homayouni, Alireza Aminlou, Miska M. Hannuksela, Moncef Gabbouj Article 68

DOI: https://doi.org/10.1145/3335053 